\title{
Adaptive Fuzzy Path Tracking Control for Mobile Robots with Unknown Control Direction
}

\author{
Qifei Du $\mathbb{D}^{1},{ }^{1}$ Lin Sha $\mathbb{D}^{2,3}$ Wuxi Shi $\mathbb{D}^{2,3}$ and Liankun Sun ${ }^{4}{ }^{4}$ \\ ${ }^{1}$ National Demonstration Center for Experimental Engineering Training Education, TianGong University, Tianjin 300387, China \\ ${ }^{2}$ Key Laboratory of Intelligent Control of Electrical Equipent, Tianjin 300387, China \\ ${ }^{3}$ School of Control Science and Engineering, Tiangong University, Tianjin 300387, China \\ ${ }^{4}$ School of Computer Science \&Technology, Tiangong University, Tianjin 300387, China \\ Correspondence should be addressed to Lin Sha; shalin@tiangong.edu.cn
}

Received 10 March 2021; Revised 13 April 2021; Accepted 21 April 2021; Published 29 April 2021

Academic Editor: Guoguang Wen

Copyright ( 2021 Qifei Du et al. This is an open access article distributed under the Creative Commons Attribution License, which permits unrestricted use, distribution, and reproduction in any medium, provided the original work is properly cited.

\begin{abstract}
In order to synthesize controllers for wheeled mobile robots (WMRs), some design techniques are usually based on the assumption that the center of mass is at the center of the robot itself. Nevertheless, the exact position of the center of mass is not easy to measure, thus WMRs is a typical uncertain nonlinear system with unknown control direction. Based on the fast terminal sliding mode control, an adaptive fuzzy path tracking control scheme is proposed for mobile robots with unknown control direction. In this scheme, the fuzzy system is used to approximate unknown functions, and a robust controller is constructed to compensate for the approximation error. The Nussbaum-type functions are integrated into the robust controller to estimate the unknown control direction. It is proved that all the signals in the closed-loop system are bounded, and the tracking error converges to a small neighborhood of the origin in a limited time. The effectiveness of the proposed scheme is illustrated by a simulation example.
\end{abstract}

\section{Introduction}

The mobile robot system is a typical nonholonomic system, and the research of tracking control has always remained one of the most challenging tasks in the area of mobile robot system.

According to whether the tracking trajectory is a function of time, tracking control is divided into trajectory tracking and path tracking. For the trajectory tracking, based on the kinematics model, the backstepping method, neural network method, fuzzy neural network method, and inputoutput linearization method were developed in [1-4]. For the path tracking, when the center of mass of the robot is exactly at the geometric center of the wheel axis, the path tracking problem was studied in [5], while the center of mass of the robot is located on the central axis of the two driving wheels, the path tracking problem was developed in [6]. The assumption in $[5,6]$ that the center of mass is located on the geometric center of the wheel axis or the central axis of the two driving wheels is a good idea for an actual operating robot system; however, the exact position of the center of mass is not easy to measure when it is actually running; thus, the mobile robot system is a typical uncertain nonlinear system. By using the universal approximation of the fuzzy system [7] and the traditional linear sliding mode control techniques, the tracking control scheme were presented for the mobile robot system with uncertainty [8-12]. However, the traditional linear sliding mode control can only achieve infinite time asymptotic convergence and cannot achieve finite time tracking. Moreover, in order to improve convergence speed, it is necessary to increase the design parameters in the sliding mode control, which can increase the gain of the controller and result in the saturation of the control input. Terminal sliding mode (TSM) [13], as an effective finite-time convergence method, has received wide attention by many scholars [14-25]. In recent years, the fast terminal sliding mode (FTSM) [18] and nonsingular Terminal sliding mode (NTSM) [19] had been applied to robot control. For example, in $[19,21,22]$, the tracking control was proposed for manipulator based on NTSM, and a simple 
terminal sliding mode control was carried out and was presented for mobile robot in [23-25]. However, the proposed methods in [23-25] were a known system whose center of mass is located at the midpoint of the drive axle. When the adaptive fuzzy system is used to control the robot, the approximation error of the fuzzy system is inevitable. In order to suppress the approximation error, it is usually necessary to introduce a robust controller [26-28]. However, in [26-28], it is necessary to assume that the control direction of the system is known. For a mobile robot system with an uncertain center of mass, the control direction is difficult to obtain. Since Nussbaum first proposed the Nussbaum-type function in 1983 and successfully solved the control of the first-order linear system with unknown control direction [29], the Nussbaum-type function has been widely used in the design of control systems [30-33]. For example, the adaptive fuzzy control was proposed for the multiinput multioutput nonlinear system with unknown control direction $[30,31]$ and for the strict feedback nonlinear system with unknown control direction [32, 33]. In this paper, the Nussbaum-type function technique is applied to the control of the mobile robot system with uncertain center of mass to solve the control problem of unknown control direction of the mobile robot caused by the uncertain center of mass.

In this paper, the path tracking control was proposed for a mobile robot with uncertain center of mass and unknown control direction. The fuzzy system is used to approximate the unknown function in the robot system, and an indirect adaptive fuzzy controller is designed by using FTSM, designing a robust controller to compensate the fuzzy approximation error, and integrating the Nussbaum-type function into the robust controller to estimate the unknown control direction. Based on the Lyapunov stability analysis, an adaptive law is designed for unknown parameters, and it is proved that this method can not only ensure that all signals in the closed-loop system are bounded but also make the tracking error converge to a small neighborhood of the origin within a finite time. The simulation results verify the effectiveness of the method in this paper.

\section{Problem Description}

The structure of the wheeled robot is shown in Figure 1. The robot has three wheels, and the front left and right wheels are driving wheels. To provide power for the vehicle body, the rear wheel is a follow-up universal wheel, which can not only move with the movement direction of the front wheel but also ensure the balance of the robot.

Because wheeled robot is affected by loads, its center of mass is usually not at the center of the robot itself. In Figure 1, it is assumed that the center of mass of the robot is at point $C$, and the midpoint of the connecting axis of the first two driving wheels of the robot is point $P$. The distance from point $P$ to point $C$ is set as $L$, and the angle between the robot's forward direction axis and the line PC is set as $\phi$. The linear velocity of the progress of robot is $u_{1}$, and the angular velocity of the robot rotation is $u_{2}$. Let the

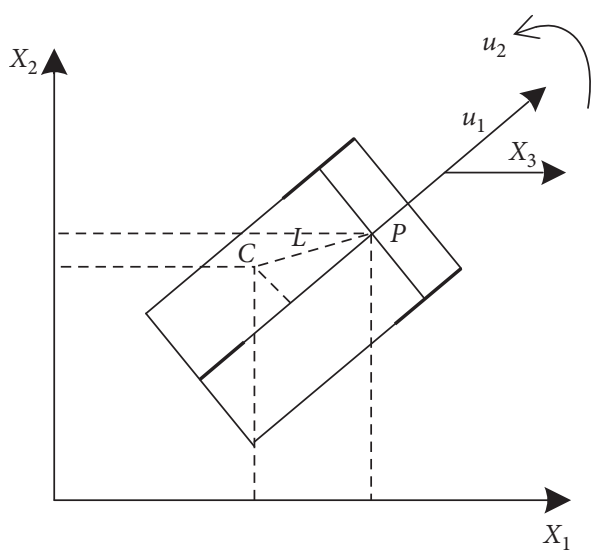

FIgURE 1: Wheeled mobile robot model.

coordinate of point $C$ as $\left(x_{1}, x_{2}, x_{3}\right)$, where $x_{1}$ is the abscissa and $x_{2}$ is the ordinate, and let the horizontal abscissa be $X_{1}$-axis, and the angle between the axis of motion direction of the vehicle body and the $X_{1}$-axis is $x_{3}$, and the $P$ coordinate is $\left(x_{1}^{\prime}, x_{2}^{\prime}, x_{3}^{\prime}\right)$; then, the kinematics equation of point $P$ is

$$
\left\{\begin{array}{l}
\dot{x}_{1}^{\prime}=u_{1} \cos x_{3}, \\
\dot{x}_{2}^{\prime}=u_{1} \sin x_{3}, \\
\dot{x}_{3}^{\prime}=u_{2} .
\end{array}\right.
$$

The location relation between point $P$ and $C\left(x_{1}, x_{2}, x_{3}\right)$ can be written as

$$
\left\{\begin{array}{l}
x_{1}=x_{1}^{\prime}-L \cos x_{3}-\phi \\
x_{2}=x_{2}^{\prime}-L \sin x_{3}-\phi \\
x_{3}=x_{3}^{\prime}
\end{array}\right.
$$

By using (1), (2) yields

$$
\left\{\begin{array}{l}
\dot{x}_{1}=u_{1} \cos x_{3}+L u_{2} \sin \left(x_{3}-\phi\right), \\
\dot{x}_{2}=u_{1} \sin x_{3}-L u_{2} \cos \left(x_{3}-\phi\right), \\
\dot{x}_{3}=u_{2},
\end{array}\right.
$$

where $\dot{x}_{1}, \dot{x}_{2}$, and $\dot{x}_{3}$ are the velocity components moving in all directions at point $C$, so (3) is the kinematics model of the robot with the center of mass $C\left(x_{1}, x_{2}, x_{3}\right)$ as the reference point.

In order to achieve the ideal control, it is assumed that path function is $f\left(x_{1}, x_{2}\right)=0$, and the tracking error function is $z=f\left(x_{1}, x_{2}\right)$; when the parameters $L$ and $\phi$ are unknown, under the effect of the amount of control $u\left(x_{1}, x_{2}, x_{3}\right)$, the system is made to move along the set path, namely, for a given value $\varphi$, there is the time $t_{1}$, and when $t>t_{1}$, the tracking error $z=f\left(x_{1}(t), x_{2}(t)\right)<\varphi$.

In this paper, it is assumed that the robot runs at linear speed $u_{1}$ and the input angular velocity $u_{2}$ is controlled. The derivative of the tracking error is

$$
\dot{z}=f_{x_{1}} \cdot \dot{x}_{1}+f_{x_{2}} \cdot \dot{x}_{2} .
$$

Substitute (3) into (4), and we have 


$$
\begin{aligned}
\dot{z}= & \left(f_{x_{1}} \cos x_{3}+f_{x_{2}} \sin x_{3}\right) u_{1} \\
& +\left(f_{x_{1}} \sin \left(x_{3}-\phi\right)-f_{x_{2}} \cos \left(x_{3}-\phi\right)\right) L u_{2}
\end{aligned}
$$

Let $\mathbf{x}=\left[x_{1}, x_{2}, x_{3}\right]^{\mathrm{T}}$ and $h(\mathbf{x})=\left(f_{x_{1}} \cdot \cos x_{3}+f_{x_{2}}\right.$. $\left.\sin x_{3}\right) u_{1}$ :

$$
g(\mathbf{x})=\left(f_{x_{1}} \cdot \sin \left(x_{3}-\phi\right)-f_{x_{2}} \cdot \cos \left(x_{3}-\phi\right)\right) L .
$$

Let $u_{2}=u$; then, (5) can be rewritten as

$$
\dot{z}=h(\mathbf{x})+g(\mathbf{x}) u .
$$

Assumption 1. There is a constant $g_{0}$ such that $0<$ $|g(\mathbf{x})|<g_{0}$.

The control law is designed as

$$
u=\frac{-h(\mathbf{x})-k z}{g(\mathbf{x})}
$$

where $\mathbf{k}>0$. Substituting (8) into (7), we obtain $\dot{z}=-\mathbf{k} z$.

Obviously, the tracking error $z$ is going to converge to zero. Since the exact position of the center of mass $C$ is unknown, that is, the distance $L$ and angle $\phi$ are unknown, the control gain $g(\mathbf{x})$ is actually an unknown nonlinear function, and it difficult to design and implement the control law (8). Considering the universal approximation of the fuzzy system, the fuzzy system is used to approximate the unknown function $g(\mathbf{x})$, and the Nussbaum-type function technique is used to estimate the unknown control direction, and the terminal sliding mode control is used to make the convergence of tracking error in a limited time.

Definition 1 (see [29]). A function $N(\zeta)$ with the following form is called Nussbaum-type function: $\lim _{s \rightarrow+\infty} \sup (1 / s)$ $\int_{0}^{s} N(\zeta) \mathrm{d} \zeta=+\infty$ and $\lim _{s \longrightarrow+\infty} \inf (1 / s) \int_{0}^{s} N(\zeta) \mathrm{d} \zeta=-\infty$. The common Nussbaum-type functions are $\zeta^{2} \cos (\zeta)$, $\zeta^{2} \sin (\zeta)$, and $\exp \left(\zeta^{2}\right) \cos ((\pi / 2) \zeta)$.

Lemma 1 (see [34]). $V(t)$ and $\zeta(t)$ are smooth functions defined in the interval $\left[0, t_{f}\right)$ and $V(t) \geq 0, \forall t \in\left[0, t_{f}\right)$, and $N(t)=\exp \left(\zeta^{2}\right) \cos ((\pi / 2) \zeta)$ is a smooth even Nussbaum-type function. If the following inequality holds, $V(t) \leq c_{0}+$ $\int_{0}^{t}(g(\tau) N(\zeta(\tau))+1) \dot{\zeta}(\tau) d \tau, \forall t \in\left[0, t_{f}\right)$, where $g(t)$ is a time-varying parameter, and $g(t) \in I=\left[l^{-}, l^{+}\right],(0 \notin I), c_{0}$ is a reasonable constant; then, $V(t), \zeta(t)$, and $\int_{0}^{t}(g(\tau)$ $N(\zeta(\tau))+1) \dot{\zeta}(\tau) d \tau$ must be bounded in $\left[0, t_{f}\right)$.

Lemma 2 (see [20]). If the continuous function $V(t)>0$ satisfies the inequality, $\dot{V}(t)+\alpha V(t)+\beta V^{(q / p)}(t) \leq 0, \forall t>t_{0}$, then $V(t)$ will converge to the equilibrium point in finite time $t_{s}$, where $t_{s} \leq t_{0}+(p / \alpha(p-q)) \ln \left(\alpha V^{1-(q / p)}\left(t_{0}\right)+\beta / \beta\right)$, where $\alpha>0, \beta>0, p$ and $q$ are odd, and $q<p$.

\section{Design of Tracking Controller}

In order to realize the tracking error convergence in a finite time, the sliding mode surface is designed as

$$
s=\dot{z}+\alpha \cdot z+\beta \cdot z^{(q / p)}=0,
$$

where $\alpha>0, \beta>0, p$ and $q$ are odd numbers, and $q<p<2 q$. For the sake of convenience, $\gamma=(q / p)$ are assigned in this paper. The designed control law is designed as

$$
u=\frac{1}{g(\mathbf{x})}\left(-h(\mathbf{x})-\alpha \cdot z-\beta \cdot z^{\gamma}\right) .
$$

Substituting (10) into (7), we obtain $\dot{z}+\alpha \cdot z+\beta \cdot z^{\gamma}=0$. According to Lemma $1, z$ will converge in a limited time. We know that the nonlinear function $g(\mathbf{x})$ is unknown in (10), so controller (10) cannot be implemented. In this paper, the fuzzy logic system is adopted to approximate the nonlinear function $g(\mathbf{x})$. The form of the fuzzy rule base is as follows: $R^{(l)}$ : if $x_{1}$ is $F_{1}^{l}$ and, ....., and $x_{n}$ is $F_{n}^{l}$, then $y$ is $G_{l}, l=1,2,3, \ldots, M$, where $F_{i}^{l}(i=1,2, \ldots, n)$ and $G^{l}$ are fuzzy sets, respectively, belonging to functions $\mu_{F^{l}}\left(x_{i}\right)$ and $\mu_{G^{l}}(y)$, both of which are Gaussian, where $M$ is fuzzy rule number. $\mathbf{x}=\left[x_{1}, x_{2}, \ldots, x_{n}\right]^{T} \in \mathbf{R}^{n}$ is the input vector of the fuzzy system, and $y \in \mathrm{R}$ is the output variable. Using singlevalued fuzzy generator, product inference rule, and central average fuzzy eliminator, the output form of the fuzzy system can be expressed as follows:

$$
\widehat{g}(\mathbf{x} \mid \theta)=\frac{\sum_{l=1}^{M} \tilde{y}^{l}\left(\prod_{i=1}^{n} \mu_{F_{i}^{l}}\left(x_{i}\right)\right)}{\sum_{l=1}^{M}\left(\prod_{i=1}^{n} \mu_{F_{i}^{l}}\left(x_{i}\right)\right)}=\boldsymbol{\theta}^{\mathrm{T}} \boldsymbol{\psi}(\mathbf{x}),
$$

where $\theta=\left[\theta_{1}, \theta_{2}, \ldots, \theta_{M}\right]^{T}$ is the adaptive variable vector and $\theta_{l}=\tilde{y}^{l}$ is the point corresponding to the maximum value of $\mu_{G^{l}} \psi(\mathbf{x})=\left[\psi_{1}(\mathbf{x}), \ldots, \psi_{M}(\mathbf{x})\right]^{T}$ is the fuzzy basis function vector, where $\psi_{l}(\mathbf{x})=\left(\prod_{i=1}^{n} \mu_{F_{i}^{l}}\left(x_{i}\right) / \sum_{l=1}^{M} \prod_{i=1}^{n}\right.$ $\left.\mu_{F_{i}^{l}}\left(x_{i}\right)\right)$, the control law (10) may have singularity problems, so the fuzzy system (11) $\widehat{g}(\mathbf{x} \mid \theta)=\theta^{T} \psi(\mathbf{x})$ is adopted to approximate the unknown function $g(\mathbf{x})$, and the equivalent control law is designed as follows:

$$
u_{\mathrm{eq}}=\frac{1}{\widehat{g}(\mathbf{x} \mid \boldsymbol{\theta})+\varepsilon \cdot \operatorname{sign}(\widehat{g}(\mathbf{x} \mid \boldsymbol{\theta}))}\left(-h(\mathbf{x})-\alpha \cdot z-\beta \cdot z^{\gamma}\right),
$$

where $\varepsilon>0, \operatorname{sign}(\hat{g}(\mathbf{x} \mid \theta))=\left\{\begin{array}{ll}1 & \widehat{g}(\mathbf{x} \mid \theta) \geq 0 \\ -1 & \widehat{g}(\mathbf{x} \mid \theta)<0\end{array}\right.$.

To compensate the approximation error of the fuzzy system, the control law is designed as follows:

$$
u=u_{\mathrm{eq}}+u_{r}
$$

where robust control $u_{r}$ will be designed later. Substituting (12) into (6), we obtain

$$
\begin{aligned}
\dot{z}= & -\alpha z-\beta z^{\gamma}-\widehat{g}(\mathbf{x} \mid \boldsymbol{\theta}) u_{\mathrm{eq}}+g(\mathbf{x}) u_{\mathrm{eq}}+g(\mathbf{x}) u_{r} \\
& -\varepsilon \cdot \operatorname{sign}(\widehat{g}(\mathbf{x} \mid \boldsymbol{\theta})) u_{\mathrm{eq}} .
\end{aligned}
$$

The optimal parameter of the adaptive vector is defined as $\theta^{*}=\arg \left\{\min _{\theta \in \Omega_{g}}\left[\sup _{x \in \mathbf{D}_{x}}|\widehat{g}(\mathbf{x} \mid \theta)-g(\mathbf{x})|\right]\right\}$, where $\mathbf{D}_{x}$ is the definition domain of input variables of the fuzzy system and $\Omega_{g}$ is the allowable set of adaptive parameter $\theta$.

Define the minimum approximation error as $\omega=$ $\widehat{g}\left(\mathbf{x} \mid \theta^{*}\right)-g(\mathbf{x})$, and define $\Phi=\widehat{\theta}-\theta^{*}$, then (14) can be written as 


$$
\begin{aligned}
\dot{z}= & -\alpha z-\beta z^{\gamma}-\Phi^{T} \psi(\mathbf{x}) u_{\mathrm{eq}}-\omega u_{\mathrm{eq}} \\
& -\varepsilon \cdot \operatorname{sign}(\hat{g}(\mathbf{x} \mid \theta)) u_{\mathrm{eq}}+g(\mathbf{x}) u_{r}
\end{aligned}
$$

Based on universal approximation theorem, $\omega$ is bounded but not easy to measure. In this paper, we assume that there is a constant $\rho^{*}>0$ so that $|\omega| \leq \rho^{*}$, due to the unknown $\rho^{*}$, define $\widehat{\delta}_{g}$ is the estimated value of $\rho^{*}$, and let $\bar{\delta}_{g}=\widehat{\delta}_{g}-\rho^{*}$.

In (15), the sign of robust control gain $g(\mathbf{x})$ is unknown, which makes it difficult to design a robust controller $u_{r}$. However, Nussbaum-type function technique is a feasible method to solve such unknown problems; thus, Nussbaumtype function $N(\zeta)=\exp \left(\zeta^{2}\right) \cos ((\pi / 2) \zeta)$ is introduced into the design of a robust controller $u_{r}$. The robust controller $u_{r}$ is designed as follows:

$$
\begin{aligned}
& u_{r}=N(\zeta) \frac{\left(\widehat{\delta}_{g}+\varepsilon\right)^{2} u_{\mathrm{eq}}^{2} z}{\left(\widehat{\delta}_{g}+\varepsilon\right)\left|u_{\mathrm{eq}} \| z\right|+\sigma^{2}}, \\
& \dot{\zeta}=\frac{\left(\widehat{\delta}_{g}+\varepsilon\right)^{2} u_{\mathrm{eq}}^{2} z^{2}}{\left(\widehat{\delta}_{g}+\varepsilon\right)\left|u_{\mathrm{eq}} \| z\right|+\sigma^{2}},
\end{aligned}
$$

where $\sigma$ is the time-varying parameter. The following parameter adaptive law is designed as

$$
\begin{aligned}
\dot{\hat{\theta}} & =\eta \psi(\mathbf{x}) u_{\mathrm{eq}} z, \\
\dot{\hat{\delta}}_{g} & =\mu\left|u_{\mathrm{eq}} \| z\right|, \\
\dot{\sigma} & =-\lambda \sigma,
\end{aligned}
$$

where $\eta>0, \mu>0, \lambda>0$, and $\sigma(0) \neq 0$.

Theorem 1. The adaptive fuzzy controller (13) and the adaptive law of unknown parameters (17)-(19) are adopted to the robot system (7); then,

(1) All signals in a closed-loop system are bounded

(2) The tracking error $z$ will converge to a small neighborhood $\left\{z \| z \mid \leq\left(w / \alpha-\alpha^{*}\right)\right\}$ of the origin in finite time $t_{s}$, where $t_{s} \leq\left(2 / \alpha_{0}\left(1-\gamma_{0}\right)\right) \ln \left(\alpha_{0} V^{\prime 1-\gamma_{0}}\left(t_{0}\right)+\right.$ $\left.\beta_{0} / \beta_{0}\right)$

Proof. (1) Consider the following Lyapunov function candidate:

$$
V=\frac{1}{2} z^{2}+\frac{1}{2 \eta} \boldsymbol{\Phi}^{\mathrm{T}} \boldsymbol{\Phi}+\frac{1}{2 \mu} \bar{\delta}_{g}^{2}+\frac{1}{2 \lambda} \sigma^{2}
$$

The derivative of (20) is

$$
\dot{V}=z \dot{z}+\frac{1}{\eta} \Phi^{\mathrm{T}} \dot{\hat{\theta}}+\frac{1}{\mu} \bar{\delta}_{g} \dot{\widehat{\delta}}_{g}+\frac{1}{\lambda} \sigma \dot{\sigma} .
$$

From (15), we have

$$
\begin{aligned}
z \dot{z}= & -\alpha z^{2}-\beta z^{\gamma+1}-\Phi^{\mathrm{T}} \psi(\mathbf{x}) u_{\mathrm{eq}} z-\omega u_{\mathrm{eq}} z \\
& -\varepsilon \cdot \operatorname{sign}(\widehat{g}(\mathbf{x} \mid \boldsymbol{\theta})) u_{\mathrm{eq}} z+g(\mathbf{x}) u_{r} z .
\end{aligned}
$$

Substituting (22) into (21), we obtain

$$
\begin{aligned}
& \dot{V}=-\alpha z^{2}-\beta z^{\gamma+1}-\boldsymbol{\Phi}^{T} \psi(\mathbf{x}) u_{\mathrm{eq}} z-\omega u_{\mathrm{eq}} z+ \\
& g(\mathbf{x}) u_{r} z-\varepsilon \cdot \operatorname{sign}(\hat{g}(\mathbf{x} \mid \boldsymbol{\theta})) u_{\mathrm{eq}} z+\frac{1}{\eta} \boldsymbol{\Phi}^{T} \dot{\hat{\theta}}+\frac{1}{\mu} \bar{\delta}_{g} \dot{\hat{\delta}}_{g}+\frac{1}{\lambda} \sigma \dot{\sigma} .
\end{aligned}
$$

Substituting (17) into (23) and because $|\omega| \leq \rho^{*}$, we obtain

$\dot{V} \leq-\alpha z^{2}-\beta z^{\gamma+1}+\left(\rho^{*}+\varepsilon\right)\left|u_{\mathrm{eq}} \| z\right|+g(\mathbf{x}) u_{r} z+\frac{1}{\mu} \bar{\delta}_{g} \dot{\hat{\delta}}_{g}+\frac{1}{\lambda} \sigma \dot{\sigma}$.

Since $\bar{\delta}_{g}=\widehat{\delta}_{g}-\rho^{*}$, we obtain

$$
\begin{aligned}
\dot{V} \leq & -\alpha z^{2}-\beta z^{\gamma+1}+\left(\widehat{\delta}_{g}+\varepsilon\right)\left|u_{\mathrm{eq}}\left\|z\left|-\bar{\delta}_{g}\right| u_{\mathrm{eq}}\right\| z\right| \\
& +g(\mathbf{x}) u_{r} z+\frac{1}{\mu} \bar{\delta}_{g} \dot{\hat{\delta}}_{g}+\frac{1}{\lambda} \sigma \dot{\sigma} .
\end{aligned}
$$

Substituting (18) into (25), we obtain

$$
\dot{V} \leq-\alpha z^{2}-\beta z^{\gamma+1}+\left(\widehat{\delta}_{g}+\varepsilon\right)\left|u_{\mathrm{eq}} \| z\right|+g(\mathbf{x}) u_{r} z+\frac{1}{\lambda} \sigma \dot{\sigma} .
$$

Substituting the robust control law (16) into (26) yields

$$
\dot{V} \leq-\alpha z^{2}-\beta z^{\gamma+1}+\left(\widehat{\delta}_{g}+\varepsilon\right)\left|u_{\mathrm{eq}} \| z\right|+\frac{1}{\lambda} \sigma \dot{\sigma}+g(\mathbf{x}) N(\zeta) \dot{\zeta} .
$$

By using (16), (27) can be rewritten as

$$
\begin{aligned}
\dot{V} \leq & -\alpha z^{2}-\beta z^{\gamma+1}+(g(\mathbf{x}(t)) N(\zeta)+1) \dot{\zeta}+\frac{1}{\lambda} \sigma \dot{\sigma} \\
& +\left(\widehat{\delta}_{g}+\varepsilon\right)\left|u_{\mathrm{eq}} \| z\right|-\frac{\left(\widehat{\delta}_{g}+\varepsilon\right)^{2} u_{\mathrm{eq}}^{2} z^{2}}{\left(\widehat{\delta}_{g}+\varepsilon\right)\left|u_{\mathrm{eq}} \| z\right|+\sigma^{2}} .
\end{aligned}
$$

Since

$$
\left(\widehat{\delta}_{g}+\varepsilon\right)\left|u_{\mathrm{eq}} \| z\right|-\frac{\left(\widehat{\delta}_{g}+\varepsilon\right)^{2} u_{\mathrm{eq}}^{2} z^{2}}{\left(\widehat{\delta}_{g}+\varepsilon\right)\left|u_{\mathrm{eq}} \| z\right|+\sigma^{2}} \leq \sigma^{2},
$$

using (28) and (29), we can obtain

$$
\dot{V} \leq-\alpha z^{2}-\beta z^{\gamma+1}+(g(\mathbf{x}(t)) N(\zeta)+1) \dot{\zeta}+\sigma^{2}+\frac{1}{\lambda} \sigma \dot{\sigma} .
$$

Substituting (19) into (29), we have 


$$
\dot{V} \leq-\alpha z^{2}-\beta z^{\gamma+1}+(g(\mathbf{x}(t)) N(\zeta)+1) \dot{\zeta} .
$$

Integrating (31), we obtain

$$
V(t)-V(0) \leq-\int_{0}^{t} \alpha z^{2}-\int_{0}^{t} \beta z^{\gamma+1}+\int_{0}^{t}(g(\mathbf{x}(\tau)) N(\zeta)+1) \dot{\zeta} \mathrm{d} \tau .
$$

The following resulted from (32):

$$
\begin{aligned}
V(t) \leq & V(0)-\int_{0}^{t} \alpha z^{2}-\int_{0}^{t} \alpha z^{2}-\int_{0}^{t} \beta\left(z^{(\gamma+1 / 2)}\right)^{2} \\
& +\int_{0}^{t}(g(\tau) N(\zeta)+1) \dot{\zeta} \mathrm{d} \tau \leq V(0)+\int_{0}^{t}(g(\tau) N(\zeta)+1) \dot{\zeta} \mathrm{d} \tau .
\end{aligned}
$$

According to Assumption $1, g(t) \in\left[-\mathrm{g}_{0}, \mathrm{~g}_{0}\right]$ and $0 \notin\left[-\mathrm{g}_{0}, \mathrm{~g}_{0}\right]$; therefore, by using Lemma $1, V(t)$, $\int_{0}^{t}(g(\tau) N(\zeta)+1) \dot{\zeta} \mathrm{d} \tau$ and $\zeta(t)$ are bounded in the interval $\left[0, t_{f}\right)$; these conclusions are also feasible at $t_{f}=+\infty$. Therefore, $z, \widehat{\theta}, \widehat{\delta}_{g}$, and $\sigma$ are also bounded. From (12) and (16), we can know that $u_{\text {eq }}$ and $u_{r}$ are also bounded. Therefore, according to (13), the control law $u$ is bounded.

(2) Since $|\omega| \leq \rho^{*}$, we get the following equation from (22):

$$
\begin{gathered}
z \dot{z} \leq-\alpha z^{2}-\beta z^{\gamma+1}+\left\|\boldsymbol{\Phi}^{\mathrm{T}}\right\|\|\psi(\mathbf{x})\|\left|u_{\mathrm{eq}} \| z\right| \\
+\left(\rho^{*}+\varepsilon\right)\left|u_{\mathrm{eq}}\|z|+| g(\mathbf{x})\| u_{r} \| z\right| .
\end{gathered}
$$

(34) can be rewritten as

$$
\begin{aligned}
z \dot{z} \leq & -z^{2}\left[\alpha-\left(\left\|\boldsymbol{\Phi}^{\mathrm{T}}\right\|\|\psi(x)\|\left|u_{\mathrm{eq}}\right|+\left(\rho^{*}+\varepsilon\right)\left|u_{\mathrm{eq}}\right|\right.\right. \\
& \left.\left.+\left|g(\mathbf{x}) \| u_{r}\right|\right)|z|^{-1}\right]-\beta z^{\gamma+1} .
\end{aligned}
$$

Let $w=\sup _{t>0}\left[\left(\left\|\Phi^{\mathrm{T}}\right\|\|\psi(\mathbf{x})\|+\rho^{*}+\varepsilon\right)\left|u_{\mathrm{eq}}\right|+\mid g(\mathbf{x})\right.$ $\left.\left\|u_{r}\right\|\right]$; then, (35) can be rewritten as

$z \dot{z} \leq-\frac{1}{2} z^{2} \cdot 2\left(\alpha-w|z|^{-1}\right)-2^{(\gamma+1 / 2)} \beta\left(\frac{1}{2} z^{2}\right)^{(\gamma+1 / 2)}$.

If $V^{\prime}=(1 / 2) z^{2}$, from (36), we obtain

$$
V^{\prime} \leq-2 \bar{\alpha}(t) V^{\prime}-2^{(\gamma+1 / 2)} \beta\left(V^{\prime}\right)^{(\gamma+1 / 2)},
$$

where $\bar{\alpha}(t)=\alpha-w|z|^{-1}$.

For any small constant $\alpha^{*}>0$, an appropriate choice of $\alpha$ is made to $\bar{\alpha}(t)=\alpha-w|z|^{-1} \geq \alpha^{*}>0$; thus, it can be obtained from equation (37) that

$$
V^{\prime} \leq-2 \alpha^{*} V^{\prime}-2^{(\gamma+1 / 2)} \beta\left(V^{\prime}\right)^{(\gamma+1 / 2)} .
$$

Equation (38) can be written as

$$
V^{\prime} \leq-\alpha_{0} V^{\prime}-\beta_{0}\left(V^{\prime}\right)^{\gamma_{0}},
$$

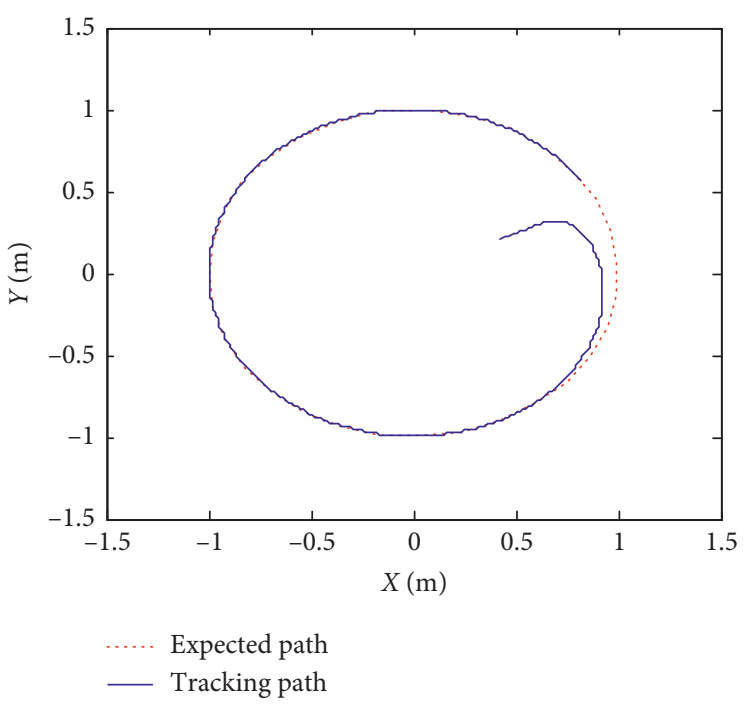

Figure 2: Expected path and tracking path.

where $\alpha_{0}=2 \alpha^{*}, \beta_{0}=2^{(\gamma+1 / 2)} \beta$, and $\gamma_{0}=(\gamma+1 / 2)$. According to Lemma 2 and $\bar{\alpha}(t)=\alpha-w|z|^{-1} \geq$ $\alpha^{*}>0$, a reasonable choice of $\alpha$ is made. Then, the tracking error $z$ will converge to a small neighborhood of the origin $\left\{z \| z \mid \leq\left(w / \alpha-\alpha^{*}\right)\right\}$ in a finite time $t_{s}$, where $t_{s} \leq\left(2 / \alpha_{0}\left(1-\gamma_{0}\right)\right) \ln \left(\alpha_{0} V^{\prime 1-\gamma_{0}}\left(t_{0}\right)+\right.$ $\left.\beta_{0} / \beta_{0}\right)$.

\section{Simulation Experiment}

The following simulation experiments will be used to verify the effectiveness of the control method designed in this paper. Let the desired path of the robot be $f\left(x_{1}, x_{2}\right)=x_{1}^{2}$ $+x_{2}^{2}-1=0$, that is, a circle with radius 1 , and set the tracking error as $z=f\left(x_{1}, x_{2}\right)=x_{1}^{2}+x_{2}^{2}-1$; then, $\dot{z}=$ $2 u_{1}\left(x_{1} \cos x_{3}+x_{2} \sin x_{3}\right)+2 L u_{2}\left[x_{1} \sin \left(x_{3}-\phi\right)-x_{2} \quad \cos \right.$ $\left.\left(x_{3}-\phi\right)\right]$. According to the above description, we have $h(\mathbf{x})=2 u_{2}\left(x_{1} \cos x_{3}+x_{2} \sin x_{3}\right)$ and $g(\mathbf{x})=2 L u_{2}\left[x_{1} \sin \right.$ $\left.\left(x_{3}-\phi\right)-x_{2} \operatorname{co}\left(x_{3}-\phi\right)\right]$.

The position of the center of mass $C$ cannot be determined from the previous description, so the control gain $g(\mathbf{x})$ is also unknown. By using the fuzzy system $\hat{g}\left(\mathbf{x} \mid \theta_{g}\right)=$ $\theta_{g}^{\mathrm{T}} \psi(\mathbf{x})$ to approximate $g(\mathbf{x})$, the membership functions inputted are as follows: $\mu_{F_{i}^{1}}\left(x_{i}\right)=\exp \left(-\left(\left(x_{i}-1.5 / 2\right)\right)^{2}\right)$, $\mu_{F_{i}^{2}}\left(x_{i}\right)=\exp \left(-\left(x_{i} / 2\right)^{2}\right), \quad$ and $\quad \mu_{F_{i}^{3}}\left(x_{i}\right)=\exp \left(-\left(\left(x_{i}+\right.\right.\right.$ $1.5 / 2))^{2}$ ), all of which are Gaussian, and $i=1,2,3$, so the fuzzy system designed in this paper has 27 rules.

In the kinematics model (3), it is assumed that $L=0.3$, $\phi=\pi / 6$, the initial attitude coordinate of the robot is $(0.4,0.2,-(\pi / 8))$, and its linear velocity $u_{1}=1$. Set the parameter in the controller $\varepsilon=0.1$, and each component of the initial value in the parameter $\hat{\theta}$ is randomly selected within the interval $[-1,1], \zeta(0)=1.2, \widehat{\delta}_{g}(0)=0.1$, and $\sigma(0)=0.1$ The parameters in the adaptive law are as follows: $\eta=3.8, \mu=0.6$, and $\lambda=0.5$.

Under the action of FTSM adaptive fuzzy controller, the robot's tracking effect on the desired path $f\left(x_{1}, x_{2}\right)=$ 


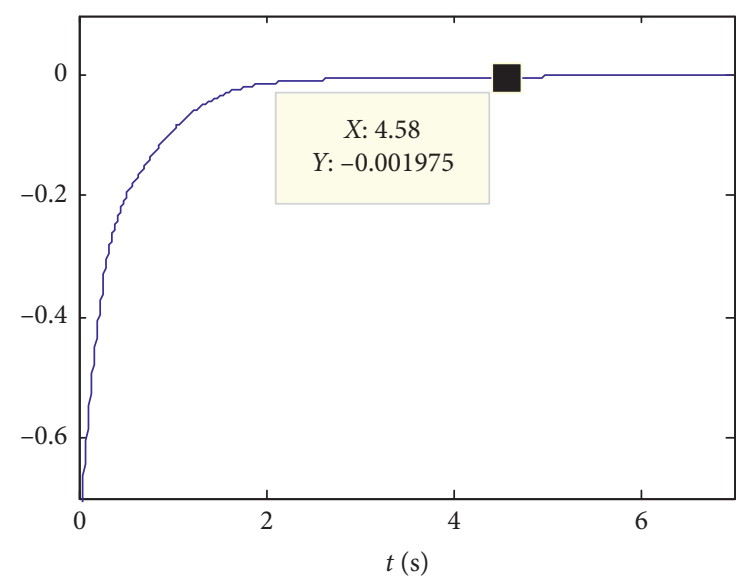

Figure 3: Error curve.

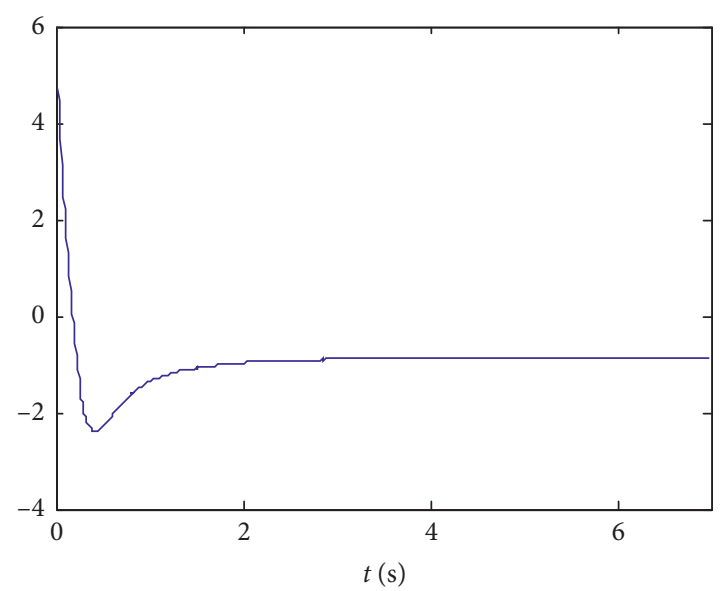

Figure 4: Controller signal.

$x_{1}^{2}+x_{2}^{2}-1=0$, namely, the circular trajectory, which is shown in Figure 2.

Figure 3 shows the error curve of robot path tracking. Figure 4 is the curve of the controller signal $u$.

From the above simulation results, it can be seen that all the signals in the closed-loop system are bounded, the error convergence of robot path tracking can be achieved, and the tracking error $z$ converges to a small neighborhood of the origin $|z| \leq 1.98 \times 10^{-3}$ after time $t_{s}=4.58 \mathrm{~s}$.

\section{Conclusion}

In this paper, an adaptive fuzzy path tracking control method has been developed for the mobile robot system with unknown mass center position. In this method, the Nussbaum-type function is used to estimate the unknown control direction, and the terminal sliding mode is used to ensure the convergence of trajectory tracking in a limited time. It is proved that the scheme can not only guarantee that all signals in the closed-loop system are bounded but also make the path tracking error of the mobile robot converge to a small neighborhood near the origin in finite time. The effectiveness of the proposed scheme is illustrated by a simulation. The research of adaptive fuzzy path tracking control method is still in the stage of theoretical research. The next step will be how to introduce engineering practice to solve practical engineering problems.

\section{Data Availability}

The data used in this article are simulated, and the authors can provide the source code for the data generation if needed.

\section{Conflicts of Interest}

The authors declare that there are no conflicts of interest regarding the publication of this paper.

\section{Acknowledgments}

This work was supported by the National Natural Science Foundation of China (51807138) and Tianjin Nature Science Foundation under Grant 20JCYBJC00180.

\section{References}

[1] Y. Kanayama, Y. Kimura, F. Miyazaki, and T. Noguchi, “A stable tracking control method for an autonomousmobile robot," in Proceedings of IEEE International Conference on Robotics and Automation, pp. 384-389, Cincinnati, OH, USA, May 1990.

[2] G. F. Yuan, S. X. Yang, and G. S. Mittal, "Tracking control of a mobile robot using a neural dynamics based approach," in Proceedings of the 2001 IEEE International Conference on Robotics and Automation, pp. 163-168, Seoul, Korea, May 2001.

[3] Y. Hu and S. X. Yang, "A fuzzy neural dynamics based tracking controller for a nonholonomic mobile robot," in Proceedings of the 2003 IEEE/ASME International Conference on Advanced Intelligent Mechatronics, pp. 205-210, Kobe, Japan, July 2003.

[4] D.-H. Kim and J.-H. Oh, "Tracking control of a two-wheeled mobile robot using input-output linearization," Control Engineering Practice, vol. 7, no. 3, pp. 369-373, 1999.

[5] B. L. Ma and W. Huo, "Path tracking control and stabilization of mobile car," Robot, vol. 17, no. 6, pp. 358-362, 1995.

[6] D. Q. Sun, W. Huo, and X. Yang, "Path following control of mobile robots with model uncertainty based on hierarchical fuzzy systems," Control Theory \& Applications, vol. 21, no. 4, pp. 489-500, 2004.

[7] L. X. Wang, Adaptive Fuzzy Systems and Control-Design and Stability Analysis, PTR Prentice-Hall, Hoboken, NJ, USA, 1994.

[8] C.-Y. Chen, T.-H. S. Li, Y.-C. Yeh, and C.-C. Chang, "Design and implementation of an adaptive sliding-mode dynamic controller for wheeled mobile robots," Mechatronics, vol. 19, no. 2, pp. 156-166, 2009.

[9] N. Yagiz and Y. Hacioglu, "Robust control of a spatial robot using fuzzy sliding modes," Mathematical and Computer Modelling, vol. 49, no. 1-2, pp. 114-127, 2009.

[10] M.-K. Chang, "An adaptive self-organizing fuzzy sliding mode controller for a 2-DOF rehabilitation robot actuated by pneumatic muscle actuators," Control Engineering Practice, vol. 18, no. 1, pp. 13-22, 2010. 
[11] R.-J. Lian, "Design of an enhanced adaptive self-organizing fuzzy sliding-mode controller for robotic systems," Expert Systems with Applications, vol. 39, no. 1, pp. 1545-1554, 2012.

[12] J. B. Hu, F. Li, G. L. Wei et al., "Theory and applications of backstepping sliding mode variable structure control for uncertain systems," Systems Engineering and Electronics, vol. 36, no. 3, pp. 519-523, 2014.

[13] M. Zak, "Terminal attractors for addressable memory in neural networks," Physics Letter A, vol. 133, no. 1-2, pp. 18-22, 1988.

[14] C. W. Tao, J. S. Taur, and M. L. Chan, "Adaptive fuzzy terminal sliding mode controller for linear systems with mismatched time-varying uncertainties," IEEE Transactions on Systems, Man and Cybernetics, vol. 34, no. 1, pp. 1-8, 2004.

[15] S. Yu, X. Yu, B. Shirinzadeh, and Z. Man, "Continuous finitetime control for robotic manipulators with terminal sliding mode," Automatica, vol. 41, no. 11, pp. 1957-1964, 2005.

[16] S. H. Yu, S. Liu, and H. Xu, "Adaptive fuzzy trajectorytracking control of uncertain nonholonomic mobile robots," in Proceedings of the 2008 6th IEEE International Conference on Industrial Informatics, pp. 13-16, Daejeon, Korea, July 2008.

[17] V. Nekoukar and A. Erfanian, "Adaptive fuzzy terminal sliding mode control for a class of MIMO uncertain nonlinear systems," Fuzzy Sets and Systems, vol. 179, no. 1, pp. 34-49, 2011.

[18] X. H. Yu and Z. H. Man, "Fast termainl sliding-mode control design for nonlinear dynamical system," IEEE Transactions on Circuits and Systems I: Fundamental Theory and Applications, vol. 49, no. 2, pp. 261-265, 2002.

[19] Y. Feng, X. Yu, and Z. Man, "Non-singular terminal sliding mode control of rigid manipulators," Automatica, vol. 38, no. 12, pp. 2159-2167, 2002.

[20] S. Yu, X. Yu, and Z. Man, "A fuzzy neural network approximator with fast terminal sliding mode and its applications," Fuzzy Sets and Systems, vol. 148, no. 3, pp. 469-486, 2004.

[21] C.-K. Lin, "Nonsingular terminal sliding mode control of robot manipulators using fuzzy wavelet networks," IEEE Transactions on Fuzzy Systems, vol. 14, no. 6, pp. 849-859, 2006.

[22] M. Jin, J. Lee, P. H. Chang, and C. Choi, "Practical nonsingular terminal sliding-mode control of robot manipulators for high-accuracy tracking control," IEEE Transactions on Industrial Electronics, vol. 56, no. 9, pp. 3593-3601, 2009.

[23] S. H. Li and Y. P. Tian, "Tracking control of mobile robots," Control and Decision, vol. 15, no. 5, pp. 626-628, 2000.

[24] S. H. Li and Y. P. Tian, "Trajectory tracking control of mobile robots in finite time," Journal of Southeast University, vol. 34, no. 1, pp. 113-116, 2004.

[25] Q. Y. Wu, M. D. Yan, and Y. Y. He, "Fast terminal sliding mode tracking controller design for nonholonomic mobile robot," Systems Engineering and Electronics, vol. 29, no. 12, pp. 2127-2130, 2007.

[26] S. Labiod, M. S. Boucherit, and T. M. Guerra, "Adaptive fuzzy control of a class of MIMO nonlinear systems," Fuzzy Sets and Systems, vol. 151, no. 1, pp. 59-77, 2005.

[27] S. Tong, B. Chen, and Y. Wang, "Fuzzy adaptive output feedback control for MIMO nonlinear systems," Fuzzy Sets Systems, vol. 156, no. 2, pp. 285-299, 2005.

[28] W. X. Shi, T. Sun, Y. X. Ma et al., "Adaptive fuzzy path following control for a nonholonomic mobile robots," in Proceedings of the 10th World Congress on Intelligent Control and Automation, WCICA, pp. 204-208, Beijing, China, July 2012.

[29] R. D. Nussbaum, "Some remarks on a conjecture in parameter adaptive control," Systems \& Control Letters, vol. 3, no. 5, pp. 243-246, 1983.

[30] A. Boulkroune, M. Tadjine, M. M'Saad et al., "Fuzzy adaptive controller for MIMO nonlinear systems with known and unknown control direction," Fuzzy Sets Systems, vol. 161, no. 6, pp. 797-820, 2010.

[31] T.-P. Zhang and Y. Yi, "Adaptive fuzzy control for a class of MIMO nonlinear systems with unknown dead-zones," Acta Automatica Sinica, vol. 33, no. 1, pp. 96-99, 2007.

[32] X. Ye and J. Jiang, "Adaptive nonlinear design without a priori knowledge of control directions," IEEE Transactions on Automatic Control, vol. 43, no. 11, pp. 1617-1621, 1998.

[33] S. S. Ge, F. Hong, and T. H. Lee, "Adaptive neural control of nonlinear time-delay systems with unknown virtual control coefficients," IEEE Transactions on Systems, Man and Cybernetics, Part B (Cybernetics), vol. 34, no. 1, pp. 499-516, 2004.

[34] W. X. Shi, "Observer-based fuzzy adaptive control for multiinput multi-output nonlinear systems with a nonsymmetric control gain matrix and unknown control direction," Fuzzy Sets and Systems, vol. 263, pp. 1-26, 2015. 